\title{
Strain and temperature sensitivity of a singlemode polymer optical fibre
}

\author{
M. Silva-Lopez ${ }^{\mathrm{a}}$, A. Fender ${ }^{\mathrm{a}}$, W.N. MacPherson ${ }^{\mathrm{a}}$, J.S. Barton ${ }^{\mathrm{a}}$, J.D.C. Jones ${ }^{\mathrm{a}}$, D. Zhao ${ }^{\mathrm{b}}$, D.J. Webb ${ }^{\mathrm{b}}$, \\ L. Zhang ${ }^{\mathrm{b}}$, I. Bennion ${ }^{\mathrm{b}}$ \\ ${ }^{a}$ School of Engineering and Physical Sciences, Heriot-Watt University, Edinburgh EH14 4AS, UK \\ ${ }^{\mathrm{b}}$ Photonics Research Group, Electronic Engineering and Computer Science Division, Aston \\ University, Birmingham B4 7ET, UK
}

\begin{abstract}
We report experimental measurements of the strain and temperature sensitivity of the optical phase in a singlemode polymer optical fibre. These values were obtained by measuring optical path length change using a Mach-Zender interferometer.
\end{abstract}

Keywords: Optical fibre sensors, polymer optical fibre, strain and temperature sensor, PMMA, Young's modulus

\section{INTRODUCTION}

Fibre optic sensors offer many advantages over conventional strain sensors. These advantages have been well documented and include insensitivity to electromagnetic fields, light weight, and minimal intrusiveness ${ }^{1}$. Fused silica, the material of choice for the majority of optical fibres, offers near ideal mechanical characteristics thus making it suitable for many strain sensing applications. However, fused silica fibres have an upper strain limit of $\sim 3-5 \%$ and in general are only reliable up to around $1 \%$ strain unless special preparation procedures are followed. In highly loaded engineering structures such as highway bridges, skyscrapers or plane wings, transverse loading can result in large bending strains, which can induce locally high strains. These can lead to the initiation of cracks and a reduction in component life, and monitoring structural strain is becoming increasingly important. With the advent of new engineering materials, such as composites, the acceptable range of applied strain can exceed the breaking strain of fused silica fibre. In such cases, standard fibre based strain gauges cannot be used. The inherent fracture toughness and flexibility of polymer optical fibers (POFs) makes them much more suitable in high strain applications than their glassbased counterparts. In addition, fibre Bragg gratings (FBG) have recently been written into POF broadening their potential applications ${ }^{2}$.

To date there have been many reports of POF sensors for strain and curvature measurement ${ }^{3}$. However, these have generally been intensiometric measurements using multimode fibres. Whilst robust sensors have been demonstrated using intensity modulating mechanisms, such sensors are always susceptible to additional intensity losses, for example bend loss, connector loss, and variations in source power. Whilst some of these may be addressed technically, by using additional reference power measurements for example, it is desirable to have a measurement mechanism that is not based upon intensity measurement. Interferometry offers a potential alternative, in which lengths of fibre can form small interferometers or can incorporate a Bragg grating. To realise these designs requires singlemode fibre rather than conventional multimode POF. The advent of singlemode POF offers the potential for high strain fibre sensors exploiting the advantages of interferometry.

\section{OPTO-MECHANICAL PROPERTIES}

The POF was supplied by Paradigm Optics and it has a cladding diameter of $125 \mu \mathrm{m}$ (commercial acrylic with $\mathrm{n}=1.4905)$ and a core diameter of $6 \mu \mathrm{m}$ (Polymethyl methacrylate (PMMA) doped with less than 3\% polystyrene with $\mathrm{n}=1.4923$ ). It is designed to be singlemode at the operating wavelength of $800 \mathrm{~nm}$ with a cut-off wavelength of $750 \mathrm{~nm}$, but in our experiments at $633 \mathrm{~nm}$ only the fundamental mode was observed. In all our experiments the scattering loss in the 
cladding and application of thermal oil/epoxy were sufficient to attenuate any cladding modes thus only the fundamental core mode contributed to the measurement.

In order to study the optical properties of the POF a Mach-Zender interferometer was set up as shown in figure 1. Light from a stabilised He-Ne laser $(633 \mathrm{~nm})$ was divided using a beamsplitter. One beam was coupled into the core of the POF, and the other travelled through free space. These were recombined using a second beamsplitter and the resultant interference was measured using a photodetector and suitable amplifier. The interferometer output was recorded on an oscilloscope from which the data could be downloaded via GPIB to a PC for further processing.

A $45 \mathrm{~cm}$ length of fibre was attached, using epoxy, to a translation stage of $\sim 10 \mathrm{~nm}$ resolution. A precision displacement sensor (LVDT) was used to independently measure the movement. A second point on the fibre was attached to a load cell. In this manner, it was possible to measure the actual phase shift (by counting the number of fringes produced) per unit strain change caused by stretching the fibre by applying a known force.

To optimise coupling in and out of the fibre, both ends of the POF were glued inside a silica capillary and the end faces polished, as shown in figure 2.

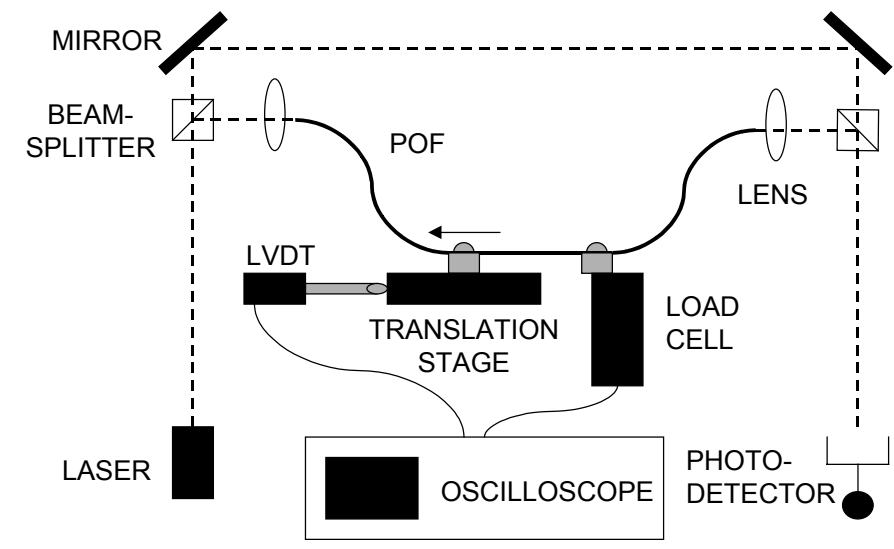

Fig 1. Setup for the strain measurement. The fibre is attached to a load cell and a translation stage, which is in contact with the displacement sensor (LVDT).

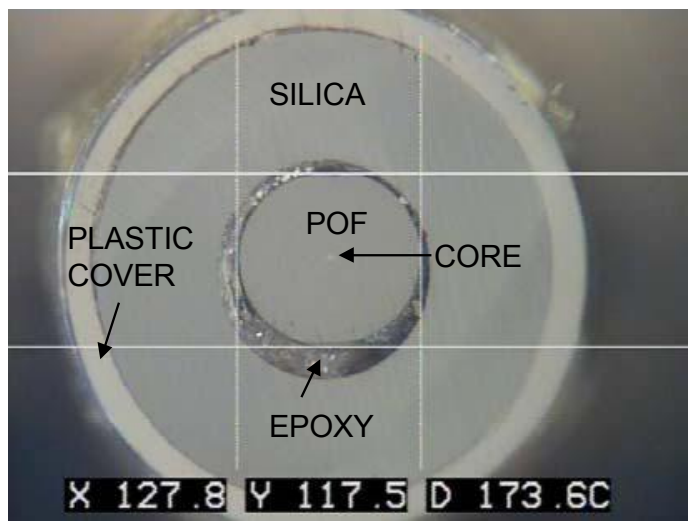

Fig 2. Polished end face of the POF glued inside the capillary.

From the measurements of the displacement and the applied force we inferred the Young's modulus to be, on average, $2.8 \pm 0.2 \mathrm{GPa}$. A plot of the applied strain versus the displacement can be seen in figure 3 ; the residuals to the linear fit are also plotted. By counting the number of fringes it was possible to measure the phase change per unit length elongation of the fibre, see figure 4 . The average value obtained was measured to be $131 \pm 3 \times 10^{5} \mathrm{rad} \mathrm{m}^{-1}$.

A simple direct comparison with theory is made difficult by the fibre drawing process, which results in molecular alignment and consequent severe anisotropy in the material parameters. As an illustration of the magnitude of this effect, we have calculated the material response using parameters appropriate to cast (isotropic) PMMA. Using first order theory of elasticity and the photoelastic effect, and assuming that the fibre is elastic and mechanically homogeneous, the phase change $d \Phi$ induced by an elongation $d L$ is given by (1) ${ }^{4}$

$$
\frac{d \Phi}{d L}=\frac{2 \pi n}{\lambda}\left\{1-\frac{n^{2}}{2}\left[p_{12}-v\left(p_{11}+p_{12}\right)\right]\right\}
$$

where $\lambda$ is the wavelength, $n$ the refractive index, $p_{\mathrm{ij}}$ the Pockels coefficients and $v$ the Poisson's ratio. For the PMMA the Pockels coefficients are $^{5} p_{11}=0.300$ and $p_{12}=0.297$ and the Poisson's ratio $^{6} v=0.34$ which gives a value $\mathrm{d} \Phi / d L=132.4 \times 10^{5} \mathrm{rad} \mathrm{m}^{-1}$ which is in good agreement with the experimental result.

Table 1 shows a comparison of the phase response $\mathrm{d} \Phi / d L$ between standard silica fibre, the fibre studied in this paper and the values predicted from theory using the coefficients for bulk PMMA. The Young's modulus is smaller for POF than for silica fiber, reflecting the high elasticity of the polymers. 

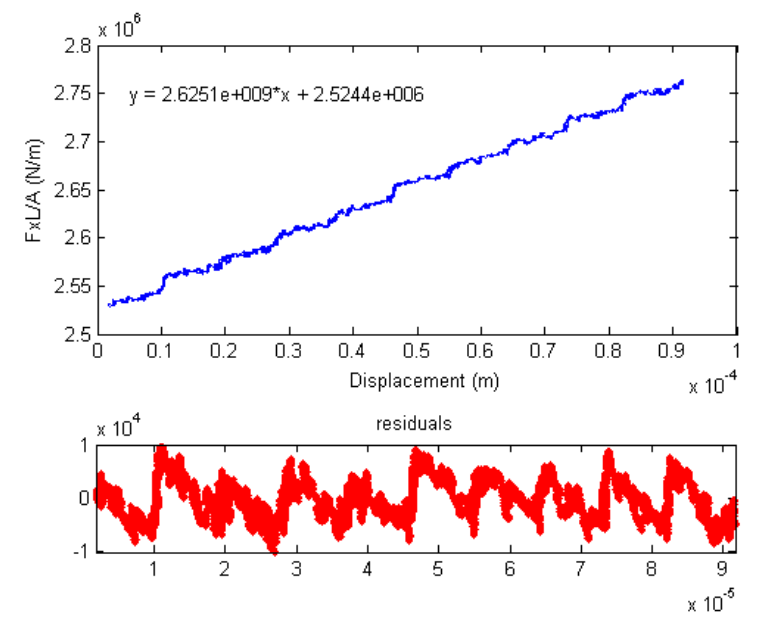

Fig. 3. The force applied to the fibre multiplied by the length and divided by the area of the fibre's cross section, versus displacement. The slope gives the Young's modulus. The residuals of the linear fit are given below.

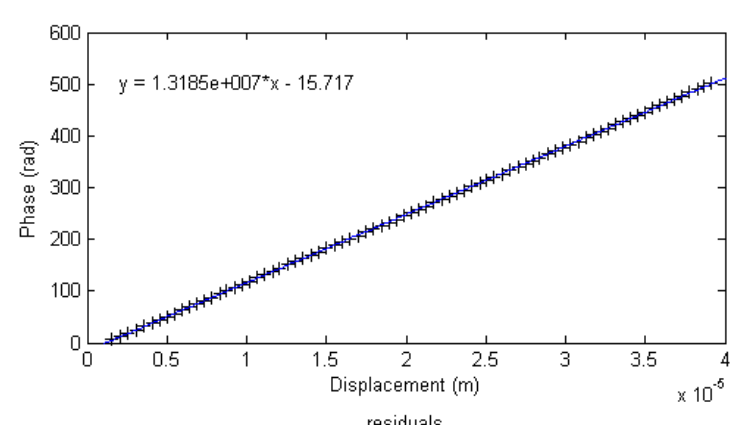

residuals

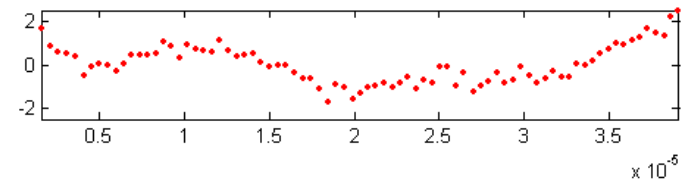

Fig. 4. The phase change versus displacement. The residuals of the linear fit are given below.

\section{THERMO-OPTIC PROPERTIES}

To determine the thermo-optic properties of the POF the same interferometer configuration was used. In this case a $32 \mathrm{~cm}$ length of fibre was fixed partially inside a high power resistor, which could be heated by applying a known current. Five holes were drilled in the ceramic surround of the resistor to locate thermocouples allowing the temperature to be monitored at several points along its $50 \mathrm{~mm}$ length. The resistor was coated with thermal oil in order to get a good thermal contact between the POF and the resistor. The resistor was connected to a power supply, which provided a constant current that could be varied to heat the resistor at different rates. This was noted to take typically between 10 and 20 seconds to heat the fibre from 20 to $40^{\circ} \mathrm{C}$. The temperature distribution closely approximated to a quadratic function, from which the fibre's average temperature was calculated by fitting data from five thermocouples (figure 5). It was observed that the fibre protruding from the edges of the resistor remained at room temperature during the timescale of the experiment. Following the same procedure as in the above section the phase was plotted as a function of the temperature (figure 6), to give a value, after normalising for fibre length, of $\mathrm{d} \Phi / d T=1111 \pm 20 \mathrm{rad} \mathrm{m}^{-1} \mathrm{~K}^{-1}$.

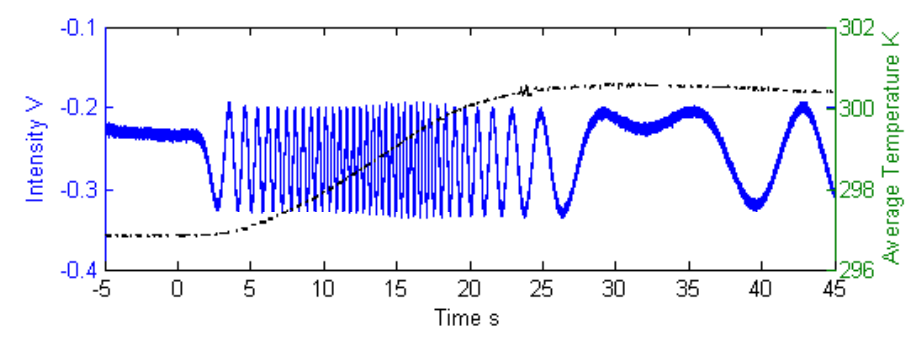

Fig. 5. Example data during fibre heating. The interference signal (solid line), and the temperature are recorded in the oscilloscope. In this case the dotted line is the average temperature calculated from five thermocouples at different positions along the heated region.

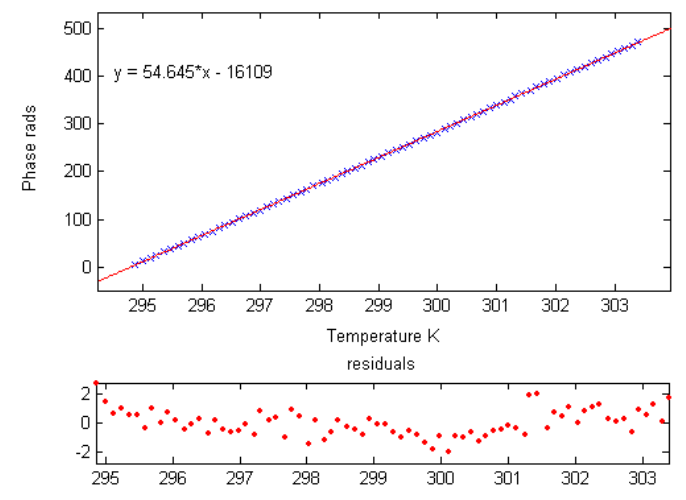

Fig. 6. Phase change versus average fibre temperature. The residuals of the linear fit are given below.

A change in fibre temperature, $d T$, produces a phase shift, $d \Phi$, of the light in the fibre. This results from a change in fibre length due to the thermal expansion and a change in the refractive index of the core (thermo-optic effect), equation (2). For PMMA, the coefficient of thermal expansion, $\alpha$, and the thermo-optic coefficient $\beta$ are ${ }^{5}$, for our range of temperature, $\alpha=70 \times 10^{-6} \mathrm{~K}^{-1}$ and $\beta=-1.2 \times 10^{-4} \mathrm{~K}^{-1}$. According to these values the phase sensitivity is calculated using 
equation (2) to be, after normalising $155.83 \mathrm{rad} \mathrm{m}^{-1} \mathrm{~K}^{-1}$. Again a comparison between the experimental and theoretical values can be seen in table 1 . It is interesting to note that, in contrast with silica, the polymers have a negative thermooptic coefficient, due to the predominant effect of the density change of the material with temperature.

$$
\frac{d \Phi}{d T}=\frac{2 \pi}{\lambda}\left[n_{o} \frac{d L}{d T}+L_{o} \frac{d n}{d T}\right]=\frac{2 \pi}{\lambda} L_{o}\left(n_{o} \alpha+\beta\right)
$$

\section{Table 1.}

Comparison between parameters of standard silica fibre, experimentally obtained from the POF, and calculated from coefficients given by the literature.

\begin{tabular}{|c|c|c|c|}
\hline & Silica fibre ${ }^{7,8}$ & POF & $\begin{array}{c}\text { Predicted from bulk } \\
\text { PMMA }\end{array}$ \\
\hline$\frac{d \Phi}{d L}$ & $115 \times 10^{5} \mathrm{rad} \mathrm{m}^{-1}$ & $131 \pm 3 \times 10^{5} \mathrm{rad} \mathrm{m}^{-1}$ & $132.4 \times 10^{5} \mathrm{rad} \mathrm{m}^{-1}$ \\
\hline$\frac{1}{L_{0}} \frac{d \Phi}{d T}$ & $99.8 \mathrm{rad} \mathrm{m}^{-1} \mathrm{~K}^{-1}$ & $1111 \pm 20 \mathrm{rad} \mathrm{m}^{-1} \mathrm{~K}^{-1}$ & $155.83 \mathrm{rad} \mathrm{m}^{-1} \mathrm{~K}^{-1}$ \\
\hline $\begin{array}{l}\text { Young's } \\
\text { modulus }\end{array}$ & $71.705 \mathrm{GPa}$ & $2.8 \pm 0.2 \mathrm{GPa}$ & $3.3 \mathrm{GPa}$ \\
\hline
\end{tabular}

\section{CONCLUSIONS}

The strain and temperature sensitivities of a PMMA polymer optical fibre have been measured experimentally and calculated from published values for bulk PMMA. For the temperature sensitivity there appears to be a considerable discrepancy between the measured fibre properties and the estimates from bulk PMMA, possibly reflecting the anisotropies introduced by the fibre drawing process. The high yield strain of polymers $(\sim 10 \%)$ is potentially advantageous in the field of interferometric fibre optic sensing in high strain applications for which silica fibre is unsuitable.

\section{ACKNOWLEDGEMENTS}

The authors would like to acknowledge the UK Engineering and Physical Science Research Council (EPSRC) and UK Defence Science and Technology Laboratory (dstl) for provision of funding through the Joint Grant Scheme, and W.N. MacPherson acknowledges the EPSRC for provision of funding via the Advanced Fellowship Programme.

\section{REFERENCES}

1. J.M. López-Higuera, Handbook of Optical Fibre Sensing Technology, Wiley, 2002.

2. H.Y. Liu, H.B. Liu, G.D. Peng, P.L. Chu, "Observation of type I and type II gratings behaviour in polymer optical fiber", Optics Communications, 220, p 337-343, 2003.

3. K.S.C. Kuang, W.J. Cantwell and P.J. Scully, “An evaluation of a novel plastic optical fibre sensor for axial strain and bend measurement”, Measurement Science and Technology, Vol. 13, p 1523-1534, 2002.

4. C.D. Butter and G.B. Hocker, "Fiber optics strain gauge", Applied Optics Vol. 17, No.18, p 2867, 1978.

5. Roy M. Waxler, Deane Horowitz and Albert Feldman, "Optical and Physical parameters of Plexiglas 55 and Lexan", Applied Optics, Vol 18, No. 1, p 101, 1979.

6. D.D. Raftopoulos, D. Karapanos and P.S. Theocaris, "Static and dynamic mechanical and optical behaviour of high polymers", Journal Physics D: Applied Physics, Vol 9, p 869, 1976.

7. A. Bertholds and R. Dandliker, "Determination of the individual strain-optic coefficients in single-mode optical fibers", Journal of Lightwave Technology, Vol. 6, No. 1, p.17, 1988.

8. B.J. White, J.P. Davis, L.C. Bobb, H.D. Krumboltz and D.C. Larson., "Optical-fiber Thermal Modulator”, Journal of Lightwave Technology, Vol. Lt 5, No. 9, p.1169, 1987.

9. J.M. Cariou, J Dugas, L. Martin and P. Michel, "Refractive-index variations with temperature of PMMA and polycarbonate", Applied Optics, Vol. 25, No.3, p. 334, 1986.

10. J. Brandrup, E.H. Immergut, E.A. Grulke, Polymer Handbook, V/87, Wiley, New York, 1999. 